\title{
Filtered Backprojection
}

National Cancer Institute

\section{Source}

National Cancer Institute. Filtered Backprojection. NCI Thesaurus. Code C94896.

A method of reconstruction that sums individual contributions to a pixel (voxel) value from detected counts of all lines of response passing through this pixel (voxel). It is an alternative approach to iterative reconstruction. Associated with this process is also a filtering that removes undesirable artifacts. 